EPJ manuscript No.

(will be inserted by the editor)

\title{
How and when can one identify hadronic molecules in the baryon spectrum
}

\author{
C. Hanhart \\ Institut für Kernphysik (Theorie), Forschungzentrum Jülich, D-52425 Jülich, Germany \\ Received: date / Revised version: date
}

\begin{abstract}
A method to identify hadronic molecules in the particle spectrum is reviewed and the conditions for its applicability discussed. Special emphasis is put on the discussion of molecule candidates in the baryon spectrum.
\end{abstract}

PACS. PACS-key discribing text of that key - PACS-key discribing text of that key

\section{Introduction}

If we want to deduce any information about quark confinement from the hadron spectrum, it is necessary to disentangle those states that exist due to inter-quark (or quark-gluon or gluon-gluon) interactions from those that exist due to hadron-hadron interactions. The latter I will call hadronic molecules, the former elementary states (sometimes I will also use the notion quark states, however, it should become clear that the method outlined only allows one to quantify the molecular component of a state and not to draw any conclusion on the composition of the elementary part, which might be a conventional meson/baryon or diquark-antidiquark/pentaquark or contain valence gluons). Since also the individual hadrons are made of quarks and gluons this distinction sounds quite academic. However, this is not the case. In this presentation I will first discuss under which circumstances we have a chance to identify hadronic molecules in a model independent way. After this, the conditions are checked for various baryons that are candidates for molecules in the spectrum. Then the example of the $\Lambda(1520)$ is discussed in some detail.

Before going into details some general remarks are useful. What we call a hadronic molecule $\mathbb{1}^{1}$ is an object that exists as the result of non-perturbative hadron-hadron interactions. For the considerations below it is not necessary to assume a particular mechanism for the hadron-hadron interaction. All that will be needed is that this interaction is attractive and sufficiently strong to form a bound state - the latter property will be parameterized by an effective coupling constant. To understand under which circumstances it is possible to disentangle hadronic molecules and quark states, let us look at the analytic properties of a general loop digram with either of these degrees of free-

\footnotetext{
1 In Ref. [1] the same objects are baptized extraordinary hadrons.
}

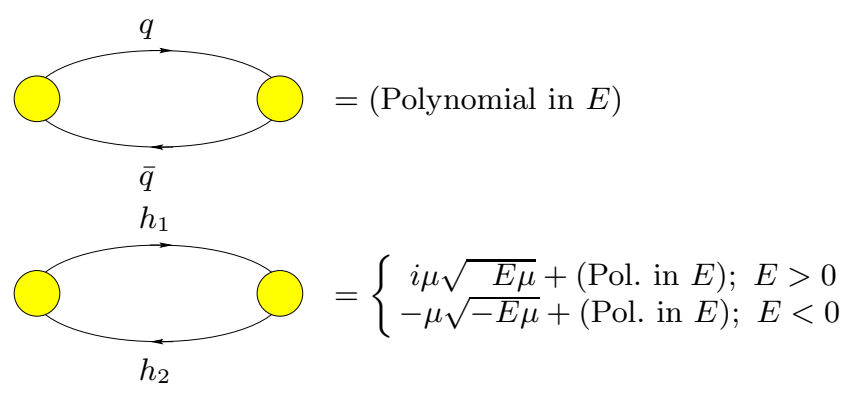

Fig. 1. Illustration of the essential difference between hadron loops (or loops of colour neutral objects) and quark-loops (or loops of coloured objects): only the former have nonanalyticities.

dom as possibilities for the essential building block of a particular state (for simplicity, in this section we discuss the case of mesons only - the generalization of the argument to baryons is straightforward). Since quarks can not go on-shell, the corresponding loop integral needs to lead to a function analytic in the energy. On the other hand, in addition to an analytic part, the hadronic loop contains also a non-analytic piece that originates from the unitarity cut. This piece is genuine to the two-hadron loop. This situation is illustrated in Fig. 1 Thus, if we can identify situations where this non-analytic piece gives the most prominent contribution for molecules, we should have a chance to disentangle model independently hadronic from quark loops or, better, hadronic molecules from elementary states. In the next section we will briefly sketch how this argument can be made quantitative.

\section{Formalism}

The analysis is based on a series of papers by S. Weinberg that allowed him to deduce that the deuteron can be 
viewed as a proton-neutron bound state 2 . The formalism can be applied, if the binding momentum $\gamma=\sqrt{2 \mu \epsilon}$ is much smaller than any other intrinsic scale of the problem. Here, $\epsilon$ denotes the binding energy with respect to the nearby continuum channel characterized by its reduced mass $\mu$. Especially, $\gamma \ll \beta$ is a necessary condition, where $\beta$ denotes the inverse of the range of forces. Then the low energy scattering parameters - or equivalently the effective coupling constant, $g_{\text {eff }}$, of the physical state to the continuum - provide a direct measure of the molecular component of the state. It was shown that (in the normalisation of Ref. [3])

$$
\begin{aligned}
\frac{g_{\text {eff }}^{2}}{4 \pi} & =16\left(m_{1}+m_{2}\right)(1-\mathcal{Z}) \sqrt{2 \mu \epsilon} \\
& \leq 16\left(m_{1}+m_{2}\right) \sqrt{2 \epsilon \mu} .
\end{aligned}
$$

where $\mathcal{Z}$ is a direct measure of the nature of the state: $\mathcal{Z}=1$ for a purely elementary state and $\mathcal{Z}=0$ for a molecule. For a short derivation of the above result see Ref. 3. In Ref. 4 it was argued that the same formalism can also be used in the presence of inelastic channels, as long as the corresponding thresholds are sufficiently far away and the states are narrow. It can be shown 4 that the results described are equivalent to those of Refs. [5] derived from the theory of scattering off a potential.

When this formalism was applied to the scalar mesons $a_{0}(980)$ and $f_{0}(980)$ it was found from an analysis of a series of reactions [9] that the latter is indeed predominantly of molecular nature, in line with the results of Refs. [10, while the former did not give results that point at an unambiguous interpretation. This might either point at a prominent non-molecular contribution to the $a_{0}$ structure, or the $a_{0}$ is not a bound state, but a virtual state. In Ref. 6] the same ideas were applied to an analysis of the decays of the $D_{s}(2317)$ viewed as a $K D$-molecule. The relation of Eq. (11) was confirmed in a microscopic model [7]. In the same spirit, in Ref. [8] the data on the hidden-charm resonance $X(3872)$ was shown to be consistent with an interpretation as a virtual state in the $D^{*} \bar{D}$ system.

In the rest of this paper we discuss the possibilities of the application of the method to the baryon spectrum, which was not yet done in detail.

\section{Application to the baryon spectrum}

Recently a sizable number of baryon resonances was identified as candidates for hadronic molecules. We will now go through some examples and check for the relevant scales of the individual problems to see to what extend the method described in the previous section is applicable.

Probably the classic example for a dynamically generated baryon state is the $\Lambda(1405)$ - proposed to originate from meson-baryon dynamics as early as 1977 [1]. It is located quite close to the $\Lambda K$ threshold $(\epsilon \sim 30 \mathrm{MeV})$, however, also the $\pi \Sigma$ threshold is nearby $(\epsilon \sim 70 \mathrm{MeV})$. In addition, recently this state was re-investigated within the chiral unitary approach [12 with the result that it is supposedly composed of two nearby singularities. This introduces a new, small parameter into the system. For these two reasons the formalism of the previous section can not be applied.

The next state of possible dynamical origin is the $\pi N$ resonance $S_{11}(1535)$ [13]. This state is known to couple strongly to the $\eta N$-channel. However, it is only a resonance in this channel since it is located above the $\eta N$ threshold $-m_{\eta}+M_{N}=1487 \mathrm{MeV}$ - and it is therefore unlikely to be a dynamical $\eta N$ state (see also Ref. 14]). In Ref. 13 the binding is provided in the strangeness channels $K \Sigma$ and $K \Lambda$ - however, those are further away from the pole position of the $S_{11}(1535)-m_{K}+M_{\Sigma}=1687$ $\mathrm{MeV}$ and $m_{K}+M_{\Lambda}=1614 \mathrm{MeV}$ - than the inelastic channel $\eta N$. Thus the method of the previous section can not be applied.

Another state that is a constant problem for quark models is the Roper resonance $P_{11}(1440)$. In Ref. [15] it was proposed as a candidate for a dynamical $\sigma N$ state - where $\sigma$ denotes the very broad lowest isoscalar meson resonance $\left(f_{0}(600)\right.$ in the particle data booklet [16]). However, due to the large width of the $\sigma$ not even a well defined threshold can be identified.

However, there are two promising candidates, namely the $\Lambda(1520)$, proposed to be a $\pi \Sigma(1385)^{*}$ bound state, and the $\Delta(1700)$, proposed to be a $\eta \Delta(1232)$ molecule (although the $K \Sigma(1385)^{*}$ channel plays a very important role) 17,18 . In what follows we will briefly discuss the former example.

The important parameters to be extracted from the data are pole positions and residues. Those can not be taken directly from the data but theory is needed for the analytic continuation from the physical axis into the complex plain where the poles are located. Thus what is needed for the analysis are not only exclusive data from various probes in many channels that allow one to fix the parameters of the theoretical models. The requirement to these models is a consistency with analyticity that the analytic continuation can be performed. The chiral unitary approach meets this requirement and therefore calculations based on it will be used for the arguments below.

Let us now focus on the $\Lambda(1520)$. Please note that the $\Sigma^{*}(1385)$ is not stable but has a decay width of $30-40$ $\mathrm{MeV}$ (depending on the charge state [16]). It is not clear how to take this into account in the relation of Eq. (1). In the following we will therefore only make a qualitative comparison with the arguments of Sec. 2 by adopting that a large effective coupling can be interpreted as an indication of a large molecular component.

In Ref. [18] the $\Lambda(1520)$ is investigated within the chiral unitary approach. The channels considered are $\pi \Sigma^{*}(1385)$ and $K \Xi^{*}(1530)$. Thus, with respect to the former channel the binding energy is only $5 \mathrm{MeV}$ (if we take the central value of the mass for the $\Sigma$ and ignore its width), while the second channel is more than $500 \mathrm{MeV}$ away. Thus the situation seems ideal. The inelastic channels of relevance are $\bar{K} N$ and $\pi \Sigma$, both more than $100 \mathrm{MeV}$ away. 
The analysis revealed 18,20, that, within the present approach, indeed the by far largest coupling of the $\Lambda(1520)$ to a continuum channel is to the $\pi \Sigma^{*}$ channel. Nevertheless the model is still consistent with a branching ratio of only $10 \%$ of the $\Lambda(1520)$ to $\pi \pi \Lambda$ ( $\Lambda \pi$ is with $88 \%$ the most important decay channel of the $\Sigma^{*}$ ), as a result of the limited phase space for $\pi \Sigma^{*}$. To test this approach it needs to be applied to various reactions. So far a fair description of the data was found for the reactions $K^{-} p \rightarrow \Lambda \pi \pi$, $\gamma p \rightarrow K^{+} K^{-} p, \gamma p \rightarrow K^{+} \pi^{0} \pi^{0} \Lambda$ and $\pi^{-} p \rightarrow K^{0} K^{-} p$ [19. In Ref. 20 an additional measurement of the reactions $p p \rightarrow p K^{+} K^{-} p$ and $p p \rightarrow p K^{+} \pi^{0} \pi^{0} \Lambda$ was proposed (the calculation needs additional input, e.g. on baryon-baryon final state interactions, and was performed on the basis of the formalism of Ref. 21)). In Ref. 22 the radiative decays $\Lambda(1520) \rightarrow \gamma \Sigma$ were studied, however, sizable discrepancies to the data were observed. Thus the model discussed so far might not be complete yet.

Eventually the model described will be improved sufficiently that it is consistent with all the available data. Then the effective coupling constants can be extracted and be compared to Eq. (1), once it is understood how to generalize this equation for unstable decay particles then a solid conclusion can be drawn on the nature of the $\Lambda(1520)$, however, already now evidence points at a prominent molecular component.

\section{Summary}

As soon as a physical state is located very close to a continuum threshold it is possible to extract information on the molecular component of that state almost directly from the data. So far this program was applied in much detail to the light scalar mesons, however, also investigations of heavier systems have started. The central result of this part was that a large effective coupling constant of a resonance to a nearby continuum channel shows a large molecular component of that state. Especially, the effective coupling constant gets maximal for a pure molecule and its value can be calculated solely in terms of the binding energy and the masses of the continuum particles.

In this note for the first time possibilities of a study of the baryon system were discussed. It is argued that the conditions for the applicability of the above mentioned formalism are not met for most of current molecule candidates. However, the $\Lambda(1520)$ and the $\Delta(1700)$, both already studied within dynamical models, were identified as promising candidates and the former was discussed in some detail. Since it is not known yet how to include a finite width of one of the continuum particles into the formalism mentioned in the previous paragraph, so far no quantitative conclusions regarding the molecular component of the $\Lambda(1520)$ can be drawn, however, qualitatively a consistent picture emerges where it appears as a state generated dynamically in the $\pi \Sigma^{*}$ channel.
2. S. Weinberg, Phys. Rev. 130, 776 (1963); 131, 440 (1963); 137 B672 (1965).

3. C. Hanhart, Eur. Phys. J. A 31 (2007) 543 arXiv:hep-ph/0609136.

4. V. Baru et al., Phys. Lett. B 586, 53 (2004).

5. D. Morgan, and M.R. Pennington, Phys. Rev. D 35, 1633 (1987); D. Morgan, Nucl. Phys. A543, 632 (1992); N. Törnqvist, Phys. Rev. D 51, 5312 (1995); M. Boglione and M.R. Pennington, Phys. Rev. D 65, 114010 (2002).

6. A. Faessler, T. Gutsche, V. E. Lyubovitskij and Y. L. Ma, Phys. Rev. D 76 (2007) 014005 arXiv:0705.0254 [hep-ph]].

7. D. Gamermann, L. R. Dai and E. Oset, arXiv:0709.2339 [hep-ph].

8. C. Hanhart, Yu. S. Kalashnikova, A. E. Kudryavtsev and A. V. Nefediev, Phys. Rev. D 76 (2007) 034007 arXiv:0704.0605 [hep-ph]].

9. C. Hanhart, Yu. S. Kalashnikova, A. E. Kudryavtsev and A. V. Nefediev, Phys. Rev. D 75 (2007) 074015 arXiv:hep-ph/0701214; Y Y Kalashnikova, A. E. Kudryavtsev, A. V. Nefediev, J. Haidenbauer and C. Hanhart, Phys. Rev. C 73 (2006) 045203 arXiv:nucl-th/0512028; Yu. S. Kalashnikova, A. E. Kudryavtsev, A. V. Nefediev, C. Hanhart and J. Haidenbauer, Eur. Phys. J. A 24 (2005) 437 arXiv:hep-ph/0412340.

10. J. Weinstein and N. Isgur, Phys. Rev. Lett. 48, 659 (1982); Phys. Rev. D 27, 588 (1983); Phys. Rev. D 41, 2236 (1990); G. Janssen et al., Phys. Rev. D 52 (1995) 2690; J. A. Oller and E. Oset, Nucl. Phys. A 620 (1997) 438 [Erratum-ibid. A 652 (1999) 407]; J. R. Pelaez, Phys. Rev. Lett. 92 (2004) 102001 arXiv:hep-ph/0309292.

11. M. Jones, R. H. Dalitz and R. R. Horgan, Nucl. Phys. B 129 (1977) 45.

12. D. Jido, J. A. Oller, E. Oset, A. Ramos and U. G. Meissner, Nucl. Phys. A 725 (2003) 181 arXiv:nucl-th/0303062.

13. N. Kaiser, T. Waas and W. Weise, Nucl. Phys. A 612 (1997) 297 arXiv:hep-ph/9607459; J. C. Nacher, A. Parreno, E. Oset, A. Ramos, A. Hosaka and M. Oka, Nucl. Phys. A 678 (2000) 187 arXiv:nucl-th/9906018; M. F. M. Lutz and E. E. Kolomeitsev, Nucl. Phys. A 700 (2002) 193 arXiv:nucl-th/0105042.

14. C. Schutz, J. Haidenbauer, J. Speth and J. W. Durso, Phys. Rev. C 57, 1464 (1998).

15. O. Krehl, C. Hanhart, S. Krewald and J. Speth, Phys. Rev. C 62 (2000) 025207 arXiv:nucl-th/9911080.

16. W. M. Yao et al. [Particle Data Group], J. Phys. G 33 (2006) 1 .

17. E. E. Kolomeitsev and M. F. M. Lutz, Phys. Lett. B 585 (2004) 243 arXiv:nucl-th/0305101;

18. S. Sarkar, E. Oset and M. J. Vicente Vacas, Nucl. Phys. A 750 (2005) 294 [Erratum-ibid. A 780 (2006) 78] arXiv:nucl-th/0407025.

19. L. Roca, S. Sarkar, V. K. Magas and E. Oset, Phys. Rev. C 73 (2006) 045208 arXiv:hep-ph/0603222.

20. L. Roca, C. Hanhart, E. Oset and U. G. Meissner, Eur. Phys. J. A 27 (2006) 373 arXiv:nucl-th/0602016.

21. C. Hanhart, Phys. Rept. $\mathbf{3 9 7}$ (2004) 155 arXiv:hep-ph/0311341.

22. M. Doring, E. Oset and S. Sarkar, Phys. Rev. C 74 (2006) 065204 arXiv:nucl-th/0601027.

\section{References}

1. R. L. Jaffe, arXiv:hep-ph/0701038. 